\title{
A Model of Methotrexate Encephalopathy: Neurotransmitter and Pathologic Abnormalities
}

Faye S. Silverstein, MD; Michael V. Johnston, MD

\begin{abstract}
Methotrexate may cause seizures, dementia, and leukoencephalopathy when given in toxic doses to children with leukemia or solid tumors. Even in therapeutic doses, treatment with this drug is associated with an increased incidence of seizures in children with leukemia. To study mechanisms of injury, juvenile rats were given multiple intraventricular injections of methotrexate and the brains were analyzed for histopathology and biogenic amine metabolites of dopamine and serotonin. Disruption of monoamine metabolism has been proposed as a cause of brain dysfunction from this chemotherapy. Multiple injections (1 or $2 \mathrm{mg} / \mathrm{kg}$ ) produced convulsions in an increasingly larger percentage of animals at higher cumulative doses, and five doses produced the neuropathological changes seen in human leukoencephalopathy. A single dose reduced the concentration of brain metabolites of dopamine, but not serotonin, six hours later. The effect was less pronounced after five doses. This rodent model should be useful for studying the metabolic basis of methotrexate encephalopathy. ( $J$ Child Neurol 1986;1:351-357)
\end{abstract}

$\mathrm{M}$ ethotrexate (Folex, Mexate), a folic acid antagonist, is commonly administered intrathecally as an integral component of the prophylaxis and treatment of acute lymphocytic leukemia and other malignancies in children. When administered in high doses alone or in combination with craniospinal irradiation, it may produce a variety of adverse central nervous system reactions. ${ }^{1-6}$ Acute reactions to high doses include seizures, myelopathy, and arachnoiditis. ${ }^{78}$ Dementia, learning disabilities, or more subtle personality changes may also develop in children after chronic treatment. ${ }^{9-14}$ An increased incidence of seizures in children treated for leukemia may be associated with the size of the methotrexate dose. ${ }^{15}$ Even in asymptomatic children, brain atrophy, focal lucencies, and intracranial calcifications are often seen on computed tomographic (CT) scans. ${ }^{16,17}$ Radiologic and pathologic studies have demonstrated that the drug

Received Dec 2, 1985. Accepted for publication Dec 31, 1985.

From the Departments of Pediatrics and Neurology, the University of Michigan Medical Center, Ann Arbor, MI.

This work was presented in 1983 at the American Academy of Neurology meeting, San Diego.

Address reprint requests to Michael V. Johnston, MD, Neuroscience Laboratory Building, University of Michigan, 1103 East Huron, Ann Arbor, MI 48104. has a propensity to injure white matter, producing a typical, predominantly periventricular leukoencephalopathy. ${ }^{18-23}$

The mechanisms causing methotrexate encephalopathy remain obscure. Methotrexate's most prominent action is to bind competitively to the enzyme dihydrofolate reductase, restricting the availability of reduced folic acid and, thus, interfering with intracellular methylation reactions ${ }^{24}$ In rapidly dividing cancer cells, the antimetabolite appears to act primarily by limiting the availability of purine and pyrimidine precursors of DNA. In the brain, dihydrofolate reductase may play an additional biochemical role in generation of reduced biopterin, the rate limiting cofactor for synthesis of the neurotransmitters dopamine and serotonin. ${ }^{25,26}$ Methotrexate may also inhibit the enzyme dihydropteridine reductase, which is the primary synthetic enzyme for biopterin. Disruption of the production of biogenic amine neurotransmitters by limiting the supply of cofactor has been suggested as a mechanism for methotrexate encephalopathy. ${ }^{25}$

To examine its effects on biogenic amine metabolism, we developed an animal model for methotrexate encephalopathy in young rats by repeated administration of intraventricular doses. We found that metho- 
trexate produced a behavioral syndrome of seizures and neuropathologic alterations resembling the histopathology seen in severe encephalopathy in humans. To examine the hypothesis that methotrexate alters biogenic amine metabolism, we studied the stable acid metabolites of dopamine and serotonin in the brain using a specific and sensitive high-performance liquid chromatographic technique.

\section{Methods}

Four-week-old male Sprague-Dawley rats (approximately $130 \mathrm{~g}$ ) were housed in individual cages, fed ad libitum, and weighed every three days. For each set of experiments, litter-mate controls exposed to identical conditions and injected with diluent alone were included.

To deliver methotrexate into the cerebral ventricular system, animals were anesthetized with either a xylazine-ketamine mixture or an Equithesin mixture (pentobarbital, chloral hydrate, magnesium sulfate, ethanol, and propylene glycol). The scalp was incised in the midline, and a 2-mm deep opening through the skull was made with a 26 -gauge needle. The hole was placed $2 \mathrm{~mm}$ lateral to the sagittal suture and $2 \mathrm{~mm}$ posterior to the coronal suture. Crystalline methotrexate without preservatives was dissolved in Elliot's B solution. Intracerebroventricular injection of drug or diluent was made to a depth of $4 \mathrm{~mm}$ from the skull surface with a Hamilton microliter syringe. The landmarks for injection were established by injecting cresyl violet suspension into the brain and examining its distribution in the ventricular system. Injection volumes ranged from 5 to $15 \mu \mathrm{L}$. After each injection, the scalp incision was closed and the animal was observed for the next hour.

Doses of methotrexate of 1 or $2 \mathrm{mg} / \mathrm{kg}$ were administered either as single doses or up to a maximum of five repeated doses given at three-day intervals. The $2 \mathrm{mg} / \mathrm{kg}$ dose was equivalent to approximately $15 \mathrm{mg} /$ $\mathrm{M}^{2}$ of rat body surface area (surface area $=0.02$ to 0.04 $\left.\mathrm{M}^{2}\right) .{ }^{27}$ For multiple doses, the same opening was used repeatedly. For each experimental group, controls received equal numbers and volumes of injection of Elliott's B solution alone.

To define histopathologic changes induced by methotrexate, five animals were injected with five doses of methotrexate $2 \mathrm{mg} / \mathrm{kg}$. Two days after the last dose, these animals and three controls were deeply anesthetized with chloral hydrate and perfused through the heart with $10 \%$ buffered formalin. The brains were removed intact, immersion fixed in $10 \%$ formalin $/ 30 \%$ sucrose for 1 week, and frozen sections
$(20 \mu \mathrm{m})$ were cut in the coronal plane. Sections were stained either for Nissl substance, for myelin with Luxol fast blue/cresyl violet, or with phosphotungstic acid and hematotoxylin (PTAH) to visualize reactive glia. The neuropathologic changes were examined in two separate sets of animals.

Three groups of animals were used for neurochemical analysis. The first group was anesthetized with the Equithesin mixture and received either one dose of methotrexate $(2 \mathrm{mg} / \mathrm{kg}$ ) or Elliot's B solution. A second group received five doses of the drug at three-day intervals. Animals in the third group were anesthetized with xylazine-ketamine and were given five doses of methotrexate $(2 \mathrm{mg} / \mathrm{kg})$. All animals were sacrificed by decapitation six hours after the last injection, and the corpus striatum was dissected out and frozen rapidly at $-70^{\circ} \mathrm{C}$. Two stable acid metabolites of dopamine, homovanillic acid (HVA) and dihydroxyphenylacetic acid (DOPAC) and the serotonin metabolite 5-hydroxyindoleacetic acid (5-HIAA) were measured concurrently using high performance liquid chromatographic electrochemical detection (HPLC-EC) ${ }^{28}$ Extracts of the corpus striatum (caudate and putamen) were prepared by homogenizing tissue in $500 \mu \mathrm{L}$ of $0.1 \mathrm{M}$ perchloric acid containing $0.1 \%$ sodium metabisulfite. One hundred fifty microliters of $1 \mathrm{M}$ Tris, $\mathrm{pH} 8.6$ was added to this suspension to enhance precipitation of particulate matter. After centrifugation at 18,000 rpm for 15 minutes, aliquots of supernatant were injected directly into the high performance liquid chromatographic system. The system included a $5 \mu, \mathrm{C}-18$ reverse phase column (Altex), and an electrochemical detector (BAS) with a glassy carbon electrode set at 0.78 volts $v \mathrm{Ag} /$ $\mathrm{AgCl}$ reference electrode. The mobile phase used was $10 \%$ methanol in water with $0.01 \mathrm{M}$ sodium acetate, $0.0001 \mathrm{M}$ EDTA, with $\mathrm{pH}$ adjusted to 3.7. Concentrations of DOPAC, HVA, and 5-HIAA were calculated by comparison of peak heights with those of external standards, and values were expressed per mg of tissue. Student's $t$-test was used to compare metabolite levels in methotrexate- and diluent-treated animals.

\section{Results}

\section{Behavior}

A single dose of $1 \mathrm{mg} / \mathrm{kg}$ of methotrexate had no obvious behavioral effects on any of the animals after recovery from anesthesia, but repetition of this dose or the administration of $2 \mathrm{mg} / \mathrm{kg}$ doses was associated with an increasing incidence of seizures and systemic toxicity (see Figure 1). The animals were irritable, jumped at a slight noise, were thinner than controls, 


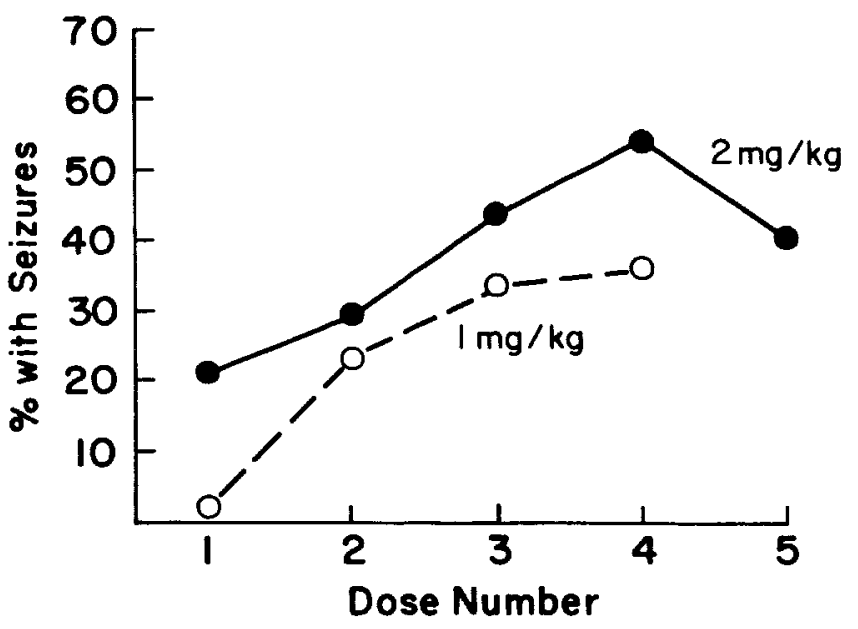

FIGURE 1

Incidence of seizures in rats after intraventricular methotrexate. Rats were given either 1 or $2 \mathrm{mg} / \mathrm{kg}$ doses of intracerebroventricular methotrexate or diluent at three-day intervals. They were lightly anesthetized with ketamine-xylazine before injection and observed for one hour afterward. Manifestations of seizures included multifocal twitching, running, clonic jerking or stiffening of body or tail. $\mathrm{N}$ at each point: 45 animals were examined after one injection; 30 after two, 20 after three, and 14 after four injections.

and were poorly groomed after they received repeated doses. The methotrexate-treated animals also appeared unsteady and tended to remain immobile much of the time. Twenty percent of the animals had obvious behavioral seizures after the first dose $(2 \mathrm{mg} / \mathrm{kg})$, while more than 50\% seized after the fourth dose (see Figure 1). Seizure manifestations included multifocal asymmetric myoclonic jerks, focal clonic face movements and body or tail stiffening, and generalized convulsions. When seizures were noted, they became apparent between 15 and 60 minutes after the injection of the drug. Seizures began when the animals were emerging from anesthesia and occurred with both types of anesthetics. Overt seizures rarely occurred for more than an hour. No controls had any behavioral manifestations of seizures.

\section{Histopathology}

The animals given five doses of $2 \mathrm{mg} / \mathrm{kg}$ methotrexate over 2 weeks had severe histopathologic changes in their brains. Foci of spongy vacuolization and gliosis were found in widespread areas and were especially severe in the corpus striatum, cortex, and periventricular white matter. Examination of myelin with Luxol fast blue (see Figure 2) showed extensive areas of fragmentation and dissolution combined with reactive gliosis. These changes were particularly obvious in periventricular areas and were present in all the animals examined after the high-dose schedule. Although controls had areas of focal inflammation near the needle tracts, none had the other histopathologic changes. The brains did not have obvious changes of hydrocephalus. The disrupted myelin and vacuolization, as well as the gliosis and loss of oligodendroglia, closely resembled the changes reported in human methotrexate-induced leukoencephalopathy. The methotrexate-treated brains also contained areas of focal neuronal necrosis in the dorsal hippocampus, striatum, and cortex.

\section{Neurochemical Analysis}

The corpus striatum was analyzed because of its heavy concentration of dopamine and serotonin terminals and its proximity to the lateral ventricles. Table 1 includes the dopamine and serotonin metabolite levels in corpus striatum after one dose or five doses of methotrexate $($ dose $=2 \mathrm{mg} / \mathrm{kg})$. A single injection of methotrexate produced a $44 \%$ decline $(p<0.001)$ in the brain concentration of DOPAC and a $30 \%$ fall $(p$

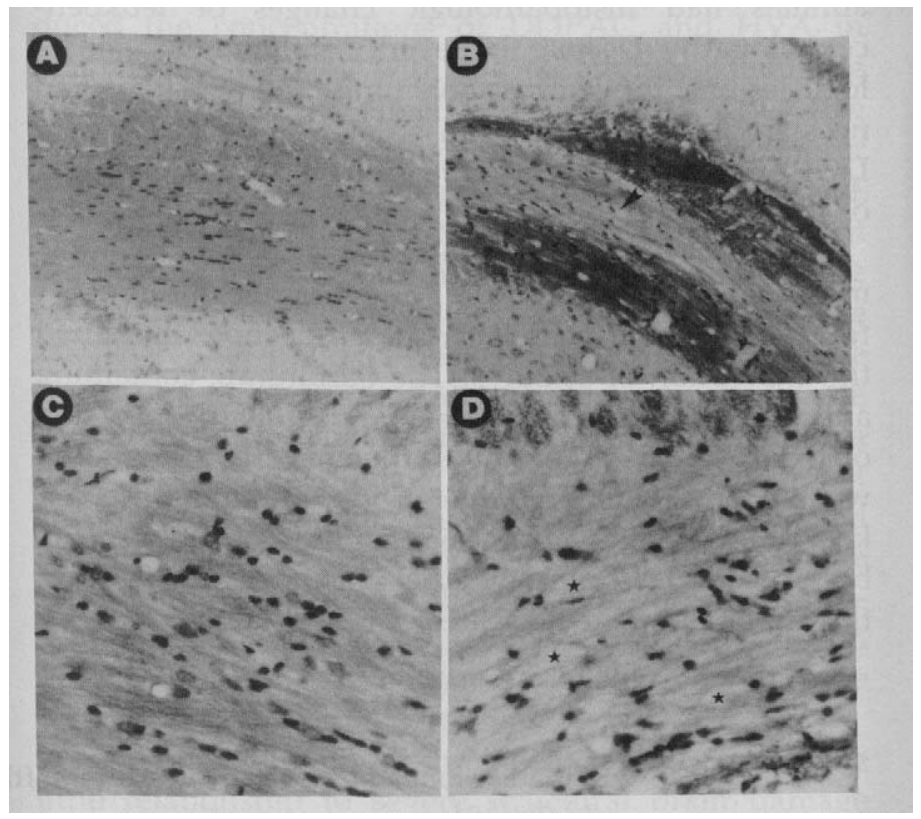

FIGURE 2

Histopathologic changes in myelin of corpus callosum of rats treated with $2 \mathrm{mg} / \mathrm{kg}$ of methotrexate every 3 days for total of 5 doses. Panels A (control) and B (methotrexate) show disruption of periventricular myelin seen in treated animals. Area at arrow in B is shown at higher power in panel D, where decreased myelin staining, vacuolization (stars) can be seen compared with panel $C$ (control). In addition, in same area there is relative paucity of oligodendroglial nuclei, and increase in reactive glia were visualized with PTAH stain (not shown). 
TABLE 1

Striatal Monoamine Metabolites After Intraventricular Methotrexate ( $2 \mathrm{mg} / \mathrm{kg}$ )Equithesin Anesthesia

\begin{tabular}{clccccccr}
\hline Dose & & N & $\begin{array}{c}5-\mathrm{HIAA} \\
(\mathrm{ng} / \mathrm{mg})\end{array}$ & $\%$ & $\begin{array}{c}\text { DOPAC } \\
(\mathrm{ng} / \mathrm{mg})\end{array}$ & $\%$ & $\begin{array}{c}\text { HVA } \\
(\mathrm{ng} / \mathrm{mg})\end{array}$ & $\%$ \\
\hline \multirow{2}{*}{1} & Control & 3 & $.67 \pm .03$ & & $.91 \pm .03$ & & $.72 \pm .01$ & \\
& Methotrexate & 5 & $.79 \pm .04$ & +15 & $.51 \pm .09^{*}$ & -44 & $.52 \pm .02^{*}$ & -28 \\
& Control & 4 & $.51 \pm .05$ & & $.77 \pm .04$ & & $.60 \pm .03$ & \\
& Methotrexate & 5 & $.80 \pm .03^{*}$ & +57 & $.61 \pm .05$ & -21 & $.60 \pm .04$ & 0 \\
\hline
\end{tabular}

Methotrexate-treated rats were given 5 doses of drug in artificial CSF every three days intracerebroventricularly and control animals were given artificial CSF alone. Before injection, they were anesthetized with Equithesin. Five hours after the last dose, the animals were sacrificed and analyzed, as described in Methods. Values expressed as mean \pm standard error of mean.

${ }^{*} p<0.001$, Student's $t$-test.

$<0.001$ ) in HVA six hours after administration, but the 5-HIAA concentration did not change. Although the single dose produced a change in neurotransmitter metabolism, it produced no obvious behavioral evidence of neurotoxicity, and a transient decline in weight gain after this dose generally was corrected in the next week (data not shown). In contrast, toxicity was noted in the animals given five consecutive doses over an approximately two-week period and these animals had histopathologic changes of leukoencephalopathy. Despite the severity of their neuropathologic lesions, the animals given multiple injections had no significant reductions in striatal levels of HVA or DOPAC. There was an increase in the striatal content of the serotonin metabolite 5-HIAA (see Table 1).

Seizures have been reported to elevate monoamine metabolites in the striatum, and the anesthesia used for the repeated intraventricular injections modified the expression of seizures. The barbiturate-containing anesthetic mixture Equithesin appeared to delay the onset of seizures and reduced their severity. Therefore, we also examined a large group of animals given ketamine-xylazine anesthesia that did not contain barbiturates to determine whether this had any effect on the monoamine metabolites. In this large group of animals, there was a modest but significant fall in HVA after five doses of methotrexate (see Table 2). The fall in DOPAC $(-16 \%)$ was not statistically significant, although it was identical to the change in HVA. In contrast to the results from experiments done with the Equithesin anesthesia mixture, 5-HIAA declined only modestly. These results suggest that a single dose of methotrexate lowered DOPAC and HVA, measures of dopamine turnover, but that with chronic administration, methotrexate's impact on dopamine metabolism is attenuated.

\section{Discussion}

Injection of repeated doses of methotrexate into the ventricular system of young rats produced seizures, systemic toxicity, and histopathologic changes characteristic of leukoencephalopathy in humans. These changes have not been studied and reported previously in a rodent model, and they provide additional evidence that methotrexate itself, without radiation, has a specific toxic effect on the brain. ${ }^{1}$

The induction of seizures confirms previous observations in humans and animals that methotrexate is an epileptogenic agent. ${ }^{7,25}$ The basis for methotrexate's

TABLE 2

Striatal Monoamine Metabolites After Fifth Dose of Intraventricular Methotrexate $(2 \mathrm{mg} / \mathrm{kg})$

\begin{tabular}{lccccccc}
\hline & $\mathrm{N}$ & $\begin{array}{c}5-\mathrm{HIAA} \\
(\mathrm{ng} / \mathrm{mg})\end{array}$ & $\%$ & $\begin{array}{c}\text { DOPAC } \\
(\mathrm{ng} / \mathrm{mg})\end{array}$ & $\%$ & $\begin{array}{c}\mathrm{HVA} \\
(\mathrm{ng} / \mathrm{mg})\end{array}$ & $\%$ \\
\cline { 2 - 7 } & 14 & $.53 \pm .06$ & & $.94 \pm .04$ & & $.79 \pm .03$ & \\
\cline { 1 - 7 } Methotrexate & 20 & $.40 \pm .05$ & -25 & $.79 \pm .08$ & -16 & $.66 \pm .04^{*}$ & -16 \\
\hline
\end{tabular}

Methotrexate-treated rats were given 5 doses of drug in artificial CSF every three days and control animals were given artificial CSF alone. Before injection, they were anesthetized with ketamine-xylazine. Five hours after the fifth dose, the animals were sacrificed and the tissue dissected rapidly for analysis, as described in Methods. Values are expressed as mean \pm standard error of mean.

${ }^{*} p<0.01$, Student's $t$-test. 
convulsant effects is uncertain. Since methotrexate contains a glutamate moiety within its structure, one possible mechanism for this action is a direct excitotoxic effect of this amino acid. ${ }^{29}$ Continued treatment was associated with an increasing incidence of seizures after later doses, suggesting that earlier brain injury occurred that sensitized the animals to the convulsive effect. Areas of focal neuronal necrosis noted in the hippocampus, striatum, and cortex may have been related to repeated seizures. These changes may parallel the pathology that underlies the increased incidence of seizures in children treated with methotrexate.

The neuropathologic changes described in patients with encephalopathy from methotrexate or methotrexate combined with radiation include spongy vacuolization of brain parenchyma, demyelination, noninflammatory necrosis of white matter, axonal swelling and necrosis, destruction of oligodendroglia, reactive gliosis, and calcification. ${ }^{18-23,30,31}$ We observed most of these changes in the methotrexate-treated rats. The brains have not yet been examined with stains specific for axonal changes. Calcification was not observed but it may not have had time to form during these short experimental periods. ${ }^{30}$ Spongy degeneration of the brain and necrosis and vacuolization of myelin were quite striking in all the brains examined. The individual doses $(2 \mathrm{mg} / \mathrm{kg}$ ) that cumulatively produced severe clinical and neuropathologic charges are high, but probably not far out of the upper range seen in clinical practice. Based on an estimate of surface area for the rats used in the experiments of $0.02-0.04 \mathrm{M}^{2}, 27$ the single dose of $2 \mathrm{mg} / \mathrm{kg}$ body weight approximated 15 $\mathrm{mg} / \mathrm{m}^{2}$. However, because of the direct administration of drug into the lateral ventricle, the brain was exposed to a much higher concentration than would occur after typical lumbar intrathecal placement. ${ }^{32}$ Nevertheless the temporally compressed, high-dose methotrexate protocol that produced changes in the rats may replicate drug exposure seen in certain clinical situations. These levels may be reached in patients with disrupted cerebrospinal fluid dynamics, those receiving direct methotrexate administration into the ventricular system, and patients who receive accidental overdoses. $7,33,34$

The results suggest that intracerebroventricular administration of methotrexate to young rats may be useful as a model for leukoencephalopathy to elucidate mechanisms of toxicity. Although clinical methotrexate toxicity involves prominent behavioral and cognitive abnormalities and seizures, virtually no information is available on the drug's effect on neuronal metabolism in the central nervous system. ${ }^{2,8,25}$ We focused on the effects of methotrexate on the stable acid metabolites of dopamine and serotonin because of the possible biochemical link between their biosynthetic pathways and biopterin cofactor. Biopterin is highly concentrated in the brain in catecholamine nerve terminals in the caudate and putamen that are relatively close to the ventricular surface. ${ }^{35}$ Biopterin required for biosynthesis of dopamine and protein is recycled by the enzyme dihydropteridine reductase, which is present in the brain. ${ }^{36}$ In vitro evidence from rat pineal glands suggests that methotrexate can reduce the concentration of biopterin at clinically relevant concentrations $(10 \mu \mathrm{mol})$ presumably by inhibiting dihydropteridine reductase ${ }^{37}$ Methotrexate inhibition of biopterin synthesis by dihydrofolate reductase could also contribute to alterations in biogenic amine metabolism, although recent in vitro data suggest that this is a minor pathway. ${ }^{38}$ If methotrexate disrupts neuronal metabolism by inhibiting biopterin production, we hypothesized that it should reduce the brain tissue levels of dopamine and serotonin metabolites. Similar changes have been observed in children with decreased $\mathrm{BH} 4$ secondary to an inherited deficiency of dihydropteridine reductase. ${ }^{39-41}$

A single injection of methotrexate significantly reduced the concentrations of DOPAC and HVA in animals anesthetized with the barbiturate-containing anesthesia, but this effect was attenuated after five injections. Repetition of the neurochemical studies after five doses of methotrexate in the nonbarbiturate anesthetized group demonstrated a small but significant reduction in HVA, but not DOPAC, which is the predominant intraneuronal dopamine metabolite in the rat. Therefore, the data provide some support for the contention that methotrexate may disrupt monoamine metabolism. The similar trends in the results from a large number of animals treated with two different anesthetics suggest that the type of anesthetic played little role. However, the modest change found in the sickest animals and the absence of a direct dose-response relationship make it unlikely that the effect on neurotransmitter metabolism is causally related to the prominent neuropathologic changes.

Although alterations in biogenic amines may have little relationship to severe structural brain damage produced by methotrexate, the early reductions suggest that this mechanism could play a role in transient behavioral changes that have been noted in certain patients. ${ }^{8-11}$ Low cerebrospinal fluid levels of HVA and 5-HIAA have been measured in some children during treatment with intrathecal methotrexate for leukemia. ${ }^{42,43}$ Although some of these children had behavioral symptoms (lassitude, headache, and anorexia), it is difficult to establish a causal relationship between 
methotrexate toxicity, low neurotransmitter metabolite levels, and transient encephalopathy. The changes could result from reduced neurotransmitter turnover or suppression of neurotransmitter catabolism. ${ }^{26} \mathrm{How}$ ever, methotrexate has not been reported to inhibit catabolic enzymes such as monoamine oxidase. Attempts to study this issue in human cerebrospinal fluid are difficult to interpret because methotrexate infusion appears to elevate cerebrospinal fluid concentrations of HVA and 5-HIAA, perhaps by blocking their egress from the cerebrospinal fluid. In leukemic children undergoing routine induction and intensification therapy that includes intrathecal methotrexate, a gradual rise in cerebrospinal fluid 5-HIAA and HVA evolves during the 9 -week period. ${ }^{43}$ Similarly, in rhesus monkeys, we found that relatively low doses of methotrexate $(0.05 \mathrm{mg} / \mathrm{kg} / \mathrm{day})$ markedly elevated cerebrospinal fluid metabolites; however, higher, toxic doses suppressed the metabolite concentrations to normal or low levels. ${ }^{34}$ Since we did not observe any increase in brain levels of these metabolites in rodents after high-dose methotrexate, these studies taken together suggest that methotrexate has distinct effects on monoamine metabolism in the brain and cerebrospinal fluid compartments.

Methotrexate is a potent neurotoxin with several possible mechanisms of action. Disruption of neurotransmitter synthesis or metabolism could account for transient encephalopathic features, but it is unlikely that this action leads to permanent neuronal damage. Leukoencephalopathy, a more serious progressive illness, is produced by several distinct therapies including methotrexate radiation and other chemotherapeutic agents such as cytosine arabinoside..$^{4-6,43,44}$ One effect shared by these treatments is their ability to inhibit protein synthesis. ${ }^{45}$ Inhibition of active protein turnover in myelin might be expected to disrupt critical structural components and oligodendroglia. The animal model may be used to examine this and other potential neurotoxic mechanisms.

\section{Acknowledgments}

This work was supported in part by Teacher Investigator Development Award NICDS NS 00603 (MVI) and a fellowship from the Canadian Medical Research Council (FSS).

Holly Arft provided expert secretarial assistance and Constance D'Amato and Samuel Hicks provided assistance with histopathological examination of the brain.

\section{References}

1. Bleyer WA: Neurologic sequelae of methotrexate and ionizing radiation: a new classification. Cancer Treat Rep 1981;65:89-97.
2. Allen JC: The effects of cancer therapy on the nervous system. J Pediatr 1978;93:903-909.

3. Kay HEM, Knopton PJ, O'Sullivan JP, et al: Encephalopathy in acute leukemia associated with methotrexate therapy. Arch Dis Child 1972;47:344-354.

4. Pizzo PA, Poplack DG, Bleyer WA: Neurotoxicities of current leukemia therapy. Am J Pediatr Hematol Oncol 1979;1:127-140.

5. Kaplan RS, Wiernik PH: Neurotoxicity of antineoplastic drugs. Semin Oncol 1982;9:103-125.

6. Hara T, Kishikawa T, Miyazaki S, et al: Central nervous system complications in childhood leukemia. Am J Pediatr Hematol Oncol 1984;6:129-136.

7. Ettinger LJ: Pharmacokinetics and biochemical effects of a fatal intrathecal methotrexate overdose. Cancer 1982;50:444-450.

8. Poplack DG: Massive intrathecal overdose: "Check the label twice!" N Engl J Med 1984;311:400-401.

9. Pizzo PA, Bleyer WA, Poplack DG, Leventhal BG: Reversible dementia temporally associated with intraventricular therapy with methotrexate in a child with acute myelogenous leukemia. J Pediatr 1976;88:131-133.

10. Ch'ien LT, Aur RJA, Stagner S, et al: Long term neurological implication of somnolence syndrome in children with acute lymphocytic leukemia. Ann Neurol 1980;8:273-277.

11. Allen JC, Rosen G: Transient cerebral dysfunction following chemotherapy for osteogenic sarcoma. Ann Neurol 1978;3:441444.

12. Pfefferbaum-Levine B, Copeland DR, Fletcher JM, et al: Neuropsychological assessment of long term survivors of childhood leukemia. Am J Pediatr Hematol Oncol 1984;6:123-128.

13. McIntosh S, Klatskin EH, O'Brien RT, et al: Chronic neurologic disturbance in childhood leukemia. Cancer 1976;37:853-857.

14. Pavlovsky $S$, Castano J, Leiiguarda $R$, et al: Neuropsychological study in patients with ALL. Am J Pediatr Hematol Oncol 1983;5: 79-86.

15. Ochs JO, Pui C, Mason C: Seizures in childhood lymphoblastic leukemia patients. Lancet 1984;2:1422-1424.

16. Peylan-Ramu M, Poplack DG, Pizzo PA: Abnormal CT scans of the brain in asymptomatic children with acute lymphocytic leukemia after prophylactic treatment of the central nervous system with radiation and intrathecal chemotherapy. $N$ Engl $J$ Med 1978;298:815-818.

17. Clausen $N$, Pederson $\mathrm{H}$ : Cranial computed tomography during treatment of childhood lymphocytic leukemia. Acta Pediatr Scand 1982;71:257-262.

18. Hendin B, Devivo D, Torack R, et al: Parenchymatous degeneration of the central nervous system in childhood leukemia. Cancer 1974;33:468-482.

19. Smith B: Brain damage after intrathecal methotrexate. J Neurol Neurosurg Psychiatr 1975;38:810-815.

20. Price RA, Jamieson PA: The central nervous system in childhood leukemia. II. Subacute leukoencephalopathy. Cancer 1975; 35:306-318.

21. Rubenstein LJ, Hermon MM, Long TF, Wilbur JR: Disseminated necrotizing leukoencephalopathy: a complication of treated central nervous system leukemia and lymphoma. Cancer 1975; 35:291-305.

22. DeVivo DC, Malas D, Nelson JS, Land VJ: Leukoencephalopathy in childhood leukemia. Neurology 1977;27:609-613.

23. Crosley CJ, Rorke LB, Evans A, Nigro M: Central nervous system lesions in childhood leukemia. Neurology 1978;28:678-685.

24. Jolivet J, Cowan KH, Curt GA, et al: The pharmacology and clinical use of methotrexate. N Engl J Med 1983;309:1094-1103.

25. Abelson HT: Methotrexate and central nervous system toxicity. 
Cancer Treat Rep 1978;62:1999-2001.

26. Johnston MV, Singer HS: Brain neurotransmitters and neuromodulators in pediatrics. Pediatr 1982;70:57-68.

27. Inglis JK: Introduction to Laboratory Animal Science and Technology. Oxford, Pergamon Press, 1980.

28. Silverstein F, Johnston M: Effects of hypoxia-ischemia on monoamine metabolism in the immature brain. Ann Neurol 1984;15: 342-347.

29. Olney JW, Fuller TA, Gubareff T: Kainate-like neurotoxicity of folates. Nature 1981;292:165-167.

30. Price RA, Birdwell DA: The central nervous system in childhood leukemia. III. Mineralizing microangiopathy and dystrophic calcification. Cancer 1978;42:717-728.

31. Shapiro WR, Allen JC, Horten BD: Chronic methotrexate toxicity to the central nervous system. Sloan-Kettering Memorial Hospital Clinical Bulletin 1980;10:49-52.

32. Bleyer WA, Dedrick RL: Clinical pharmacology of intrathecal methotrexate. I. Pharmacokinetics in nontoxic patients after lumbar injection. Cancer Treat Rep 1977;61:703-708.

33. Bleyer WA, Drake JC, Chabner BA: Neurotoxicity and elevated cerebrospinal fluid methotrexate concentration in meningeal leukemia. N Engl J Med 1973;289:770-773.

34. Johnston MV, Silverstein FS, Greenberg HS, et al: Intraventricular methotrexate alters cerebrospinal fluid homovanillic acid levels in rhesus monkeys. Neurology 1983;33(suppl 2):109.

35. Levine RA, Miller LP, Lovenberg W: Tetrahydrobiopterin in striatum: Localization in dopamine nerve terminals and role in catecholamine synthesis. Science 1981;214:919-921.
36. Pollock RJ, Kaufman S: Dihydrofolate reductase is present in brain. J Neurochem 1977;30:253-256.

37. Culvenor AJ, Miller LP, Levine RA, Lovenberg W: Effects of methotrexate on biopterin levels and synthesis in rat cultured pineal glands. J Neurochem 1984;42:1707-1714.

38. Liang BT, Vaccaro KK, Perelle BA, Perlman RL: Studies on dihydropteridine reductase activity in pheochromocytoma cells. J Neurochem 1981;37:1164-1169.

39. Koslow SH, Butler IJ: Biogenic amine synthesis defect in dihydropteridine reductase deficiency. Science 1977;198:522-523.

40. Kaufman S, Berlow S, Summer GK, et al: Hyperphenylalaninemia due to a deficiency of biopterin. N Engl J Med 1978;299:673679.

41. Brewster TG, Moskowitz MA, Kaufman S: Dihydropteridine reductase deficiency associated with severe neurologic disease and mild hyperphenylalaninemia. Pediatr 1979;63:94-99.

42. Shaywitz BA, Cohen DJ, Bowers MB: Cerebrospinal fluid monoamine metabolites in neurological disorders of childhood, in Wood JH (ed): Neurobiology of Cerebrospinal Fluid. New York, Plenum Publishing Corp, 1980.

43. Silverstein FS, Hutchinson RJ, Johnston MV: Cerebrospinal fluid biogenic amine metabolism in children during treatment for acute lymphoblastic leukemia. Pediatr Res 1986;20:285-291.

44. Winkelman MD, Hines JD: Cerebellar degeneration caused by high-dose cytosine arabinoside: A clinicopathological study. Ann Neurol 1983;14:520-527.

45. Gilman AG, Goodman LS, Gilman A: The Pharmacological Basis of Therapeutics. New York, MacMillan Inc, 1980, pp 1272-1274. 